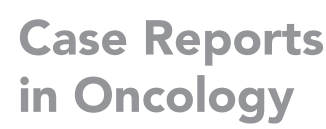

\title{
Transient Pleural Fluid Infiltration by Clonal Plasma Cells Associated with Pulmonary Tuberculosis
}

\author{
Dina Sameh Soliman a, d, e Mohammad Alib Susanna Akikkia, e \\ Feryal Ibrahim $^{a}$ Halima El-Omaric $^{\mathrm{c}}$ Ahmad Al-Sabbagh ${ }^{\mathrm{a}, \mathrm{e}}$ Lina Okar $^{\mathrm{b}}$ \\ aDepartment of Laboratory Medicine and Pathology, National Center for Cancer Care and \\ Research, Hamad Medical Corporation, Doha, Qatar; ${ }^{b}$ Department of Medical Education,

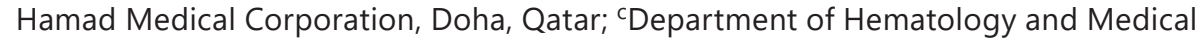 \\ Oncology, National Center for Cancer Care and Research, Hamad Medical Corporation, \\ Doha, Qatar; ${ }^{\mathrm{d} D e p a r t m e n t}$ of Clinical Pathology, National Cancer Institute, Cairo University,

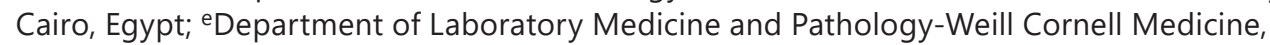 \\ Doha, Qatar
}

\section{Keywords}

Myelomatous pleural effusion · Acute myeloid leukemia · Plasma cell myeloma - Monoclonal gammopathy of undetermined significance $\cdot$ Tuberculosis

\begin{abstract}
Pleural effusion is a rare presentation of plasma cell myeloma, occurring in around $6 \%$ of patients during the course of their disease, most commonly as a consequence of a concurrent disease process like heart failure secondary to amyloid deposition. Direct infiltration of the pleural fluid by malignant cells leading to myelomatous pleural effusion is a rare mechanism occurring in less than $1 \%$ of patients with plasma cell myeloma, and it is associated with a worse prognosis. There are few case reports of myelomatous pleural effusion as an initial presentation of multiple myeloma. Pleural fluid infiltration by monoclonal plasma cells in the absence of an underlying plasma cell myeloma was not reported before in the literature. Tuberculosis is a known cause of polyclonal gammaglobulinemia, however few case reports described the coexistence of monoclonal gammopathy of undetermined significance and tuberculosis. Here we present an interesting case of pleural fluid infiltration by an abnormal looking clonal plasma cells associated with active pulmonary tuberculosis and parapneumonic effusion in a patient with a background of acute myeloid leukemia. Interestingly, the clonal plasma cell proliferation was confined to the pleural fluid without any evidence of an underlying plasma cell neoplasms (including monoclonal gammopathy of undetermined significance and plasmacytomas). Since our patient had an underlying meyloid neoplasm, we though about the possibility of secondary malignancy. However, in almost all patients with coexisting
\end{abstract}


myeloid and plasma cell neoplasms, myeloid neoplasms developed following chemotherapeutic treatment of plasma cell neoplasms not the other way around. Given that, one must conclude localized extramedullary (pleural) plasma cell proliferation probably represents a transient reactive process to pulmonary tuberculosis which is an extremely rare phenomenon and not described before.

(C) 2020 The Author(s).

Published by S. Karger AG, Basel

\section{Introduction}

Plasma cell myeloma (PCM) is a malignant neoplasm of plasma cells which proliferates and produces monoclonal immunoglobulin. It accounts for $1-2 \%$ of all cancers and around $17 \%$ of hematological malignancies in the United States [1].

The diagnosis of PCM requires the demonstration of at least $10 \%$ clonal plasma cells in the bone marrow (BM) or soft tissue plasmacytoma, and the presence of related organ impairment often recalled by the abbreviation CRAB, which stands for increased calcium level, renal insufficiency, anemia, and bone lesions, or the presence of biomarkers associated with near-inevitable progression to end organ damage like the presence of more than $60 \%$ of clonal plasma cells in the BM [2].

Monoclonal gammopathy of undetermined significance (MGUS) is a premalignant condition of plasma cells with serum monoclonal protein at a concentration of less than $3 \mathrm{~g} /$ $\mathrm{dL}$, a BM with less than $10 \%$ of monoclonal plasma cells, and absence of end organ damage. It has been described in association with many conditions including B-cell lymphoproliferative disorders, connective tissue disorders, hepatitis C, HIV, and rarely mycobacterium tuberculosis (TB) infection [3].

There is a well-documented association between PCM and other malignancies, particularly hematological malignancies. A study done on 600 patients with PCM found $10 \%$ to have another malignancy. One third of the patients developed the other malignancy subsequent to PCM diagnosis and two thirds of the patients had an underlying malignancy when diagnosed with multiple myeloma (MM). The most common subsequent type of malignancy is acute myeloid leukemia (AML) and myelodysplastic syndrome (MDS), which is a well-recognized complication of cancer therapy. Solid tumors are more frequently detected before or during the course of MM diagnosis, unlike hematological malignancies, which usually develop secondary and are mostly therapy related [4].

Pleural effusion is an uncommon manifestation of PCM, which usually happens later in the disease course, and it usually occurs due to a concurrent disease process. The most common mechanism that leads to pleural effusion in patients with PCM is heart failure. Infiltration of pleural fluid by malignant plasma cells leading to myelomatous pleural effusion (MPE) is a rare mechanism and is associated with a worse prognosis. It is important to note that it is rare for patients with PCM to present initially with MPE [5]. Diagnostic criteria to confirm the myelomatous etiology are the detection of atypical plasma cells in pleural fluid cytology, demonstration of the monoclonal protein in pleural fluid electrophoresis, which is equivalent to serum protein electrophoresis, and finally histologic confirmation with biopsy [6].

Here we present an interesting case of relapsed AML complicated by pulmonary TB and parapneumonic pleural effusion. Routine morphologic examination of pleural fluid showed many aggressive looking plasma cells including some forms with plasmablastic morphology. Flow cytometry (repeated twice) revealed an abnormal population of monotypic plasma cells confirmed by molecular studies. However, a thorough workup to exclude PCM was negative. The patient was followed up and chest X-ray repeated 1 and 3 months later showed complete 
resolution of the pleural effusion with absence of BM pathology, lytic bony lesions or masses elsewhere.

Two possible explanations for this finding were brought into discussion: the first is that pleural fluid infiltration could be an initial presentation of PCM developed as a secondary (therapy-related) neoplasm and the second less likely hypothesis is that this is a reactive clonal plasma cell proliferation in response to pulmonary TB. Negative myeloma workup repeated upon follow-up and resolved pleural effusion after treatment of TB had supported the latter possibility. This is the first case of transient clonal plasma cell proliferation as an unusual transient (reactive) process in response to pulmonary TB.

\section{Case Presentation}

A 31-year-old Ugandan male patient, with a background of acute monocytic leukemia and 11q23 deletion, stratified as high risk, received two inductions and three consolidations after which he went into clinical remission. Ten months later, he developed a relapse; BM examination showed AML with monocytic features, in addition to the development of myeloid sarcoma in the skin with similar immunophenotype and cytogenetics as the original disease. He was started on salvage chemotherapy with FLAG-IDA, and developed febrile neutropenia with high-grade fever reaching $39.2^{\circ} \mathrm{C}$. Septic workup did not reveal a focus of infection. Despite broad-spectrum antimicrobial and antifungal treatment, the patient started to deteriorate with severe respiratory distress, chest pain, dyspnea, abdominal pain, nonsubsiding high-grade fever, electrolyte imbalance, and severe hypoalbuminemia. Chest X-ray was done and it showed a right mid-zone consolidation with significant right-side pleural effusion (Fig. 1). He was further investigated with a pan-CT scan, which showed a massive pneumonic consolidation in the lower lobe of the right lung associated with considerable pleural effusion. Bronchoscopy was done and bronchoalveolar lavage was positive for mycobacterium TB.

Pleural tapping analysis showed lymphocytic exudative fluid. Cytospin preparation showed many mature looking plasma cells, some pleomorphic atypical looking plasma cells

Fig. 1. CXR showing significant right pleural effusion with underling consolidation.

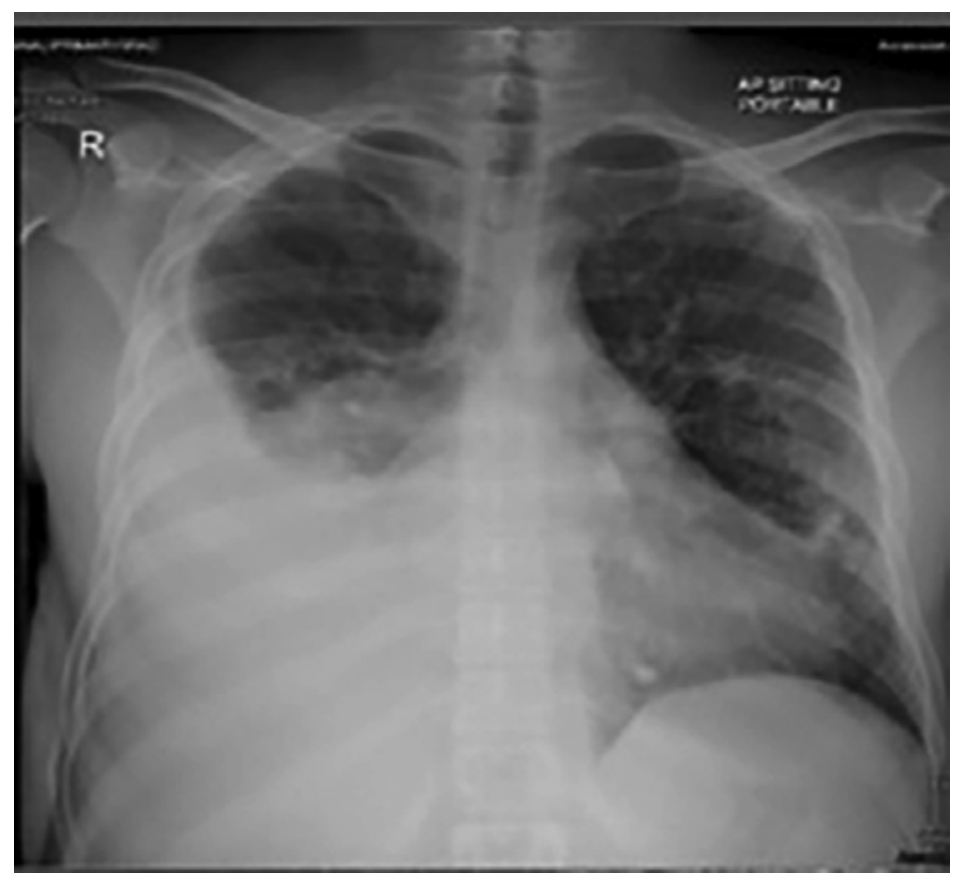



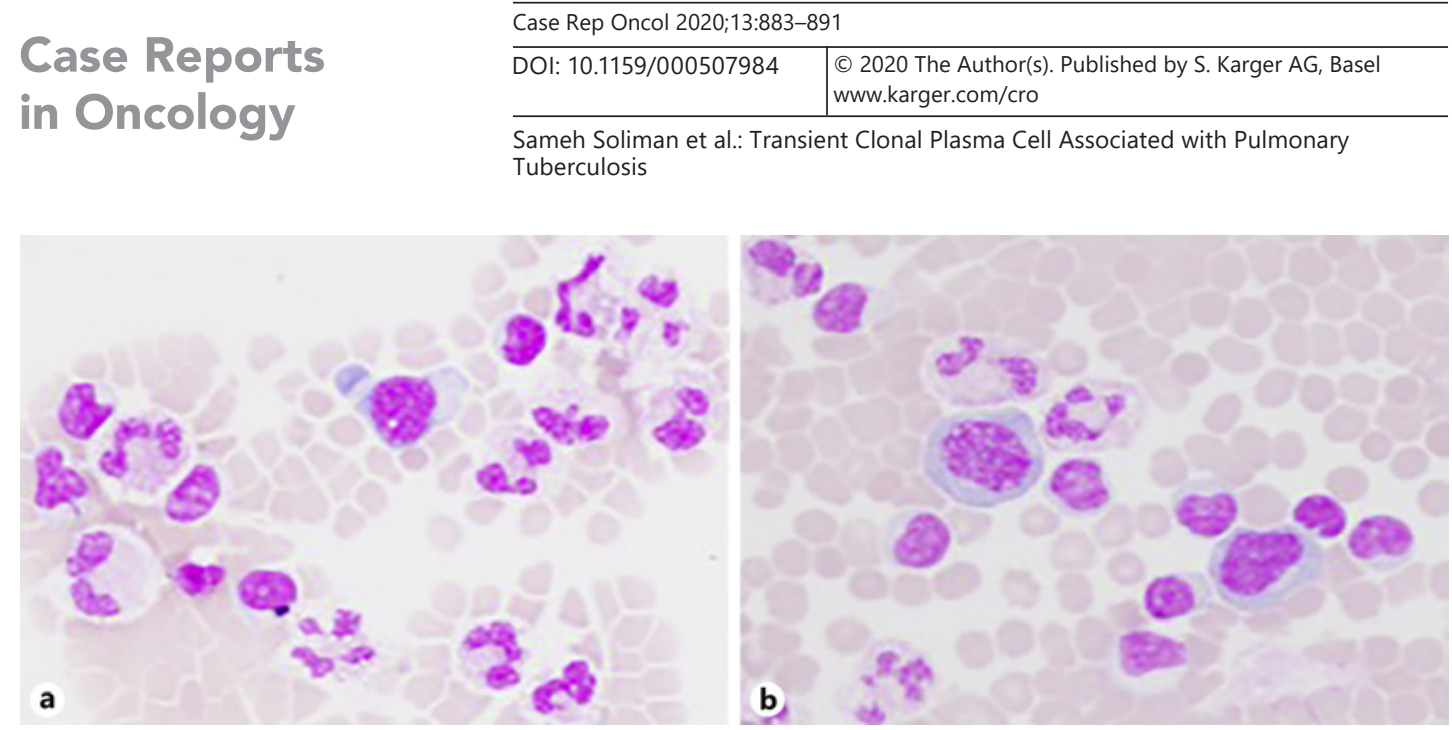

Fig. 2. Cytospin preparation is cellular and shows many red blood cells, many neutrophils and many lymphocytes. There are some abnormal looking plasma cells seen (larger with less clumped nuclear chromatin and few nucleoli).

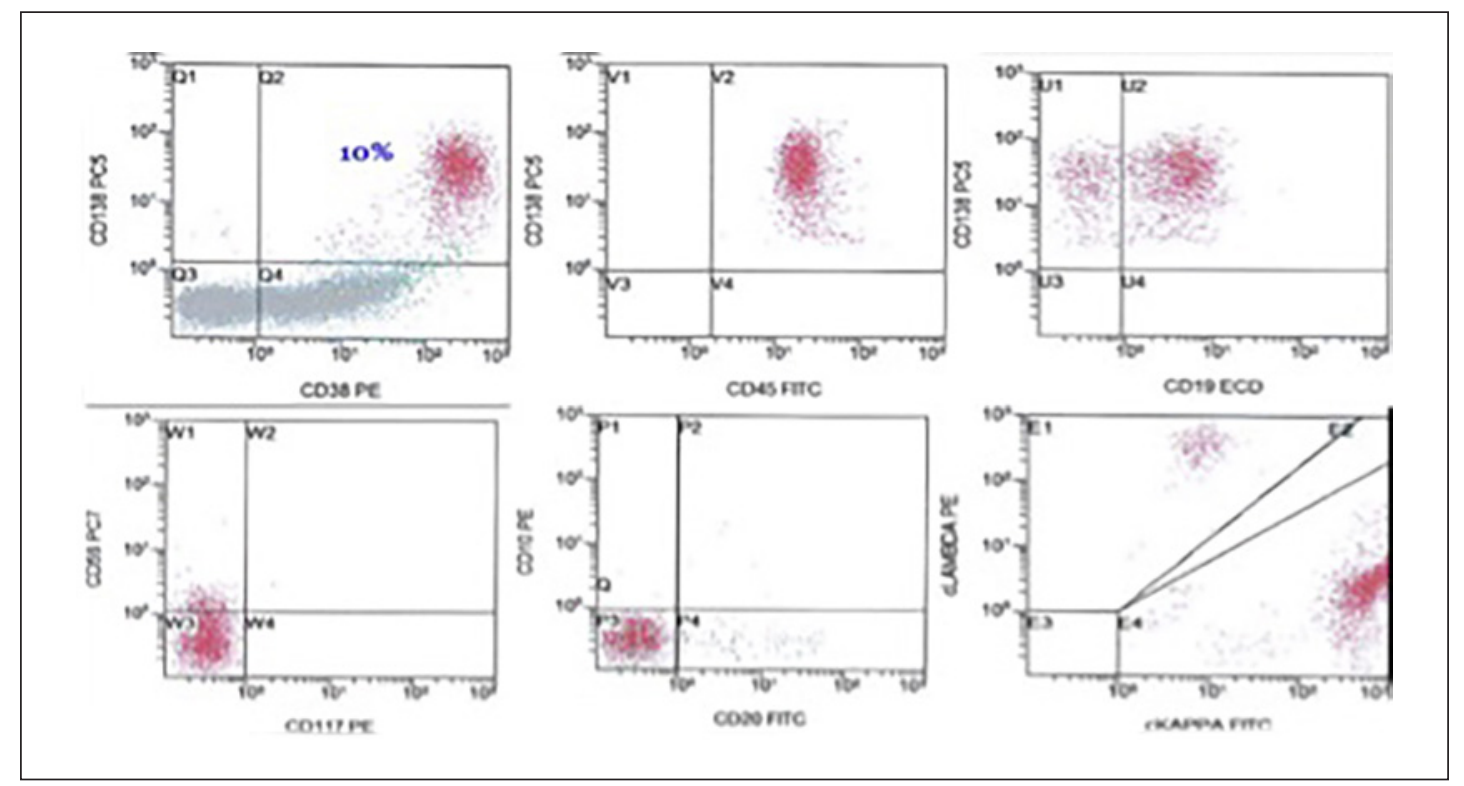

Fig. 3. Flow cytometry on pleural fluid shows an abnormal population of monotypic plasma cells ( 10\%), expressing CD138, CD45, and CD19 with cytoplasmic kappa light chain restriction.

(with multinucleation), few forms with plasmablastic morphology (larger with less clumped nuclear chromatin and few nucleoli) and few anaplastic forms (with marked nuclear irregularities) and increased mitotic figures (Fig. 2). Because of the abnormal morphology, flow cytometry was carried out to assess the phenotype of these cells. It revealed an abnormal population of monotypic plasma cells ( $\sim 10 \%)$ expressing CD45, CD38, CD138, and CD19 (majority) and showed cytoplasmic kappa light chain restriction. Plasma cells were negative for CD117 and CD20 with no significant expression of CD56 (Fig. 3).

Due to the unusual finding that was not supported by any other clinical, radiologic or biochemical evidence, flow cytometry was repeated, and it revealed the same finding. Furthermore, clonality was confirmed by PCR analysis of the conserved framework regions of the immunoglobulin heavy chain variable and joining regions (Fig. 4), which revealed clonal B-cell population without doubt. 

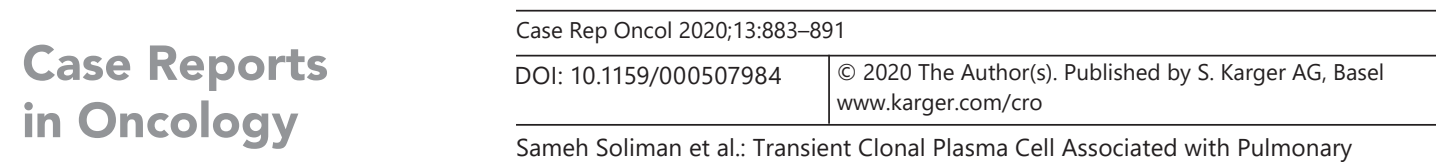

Sameh Soliman et al.: Transient Clonal Plasma Cell Associated with Pulmonary Tuberculosis

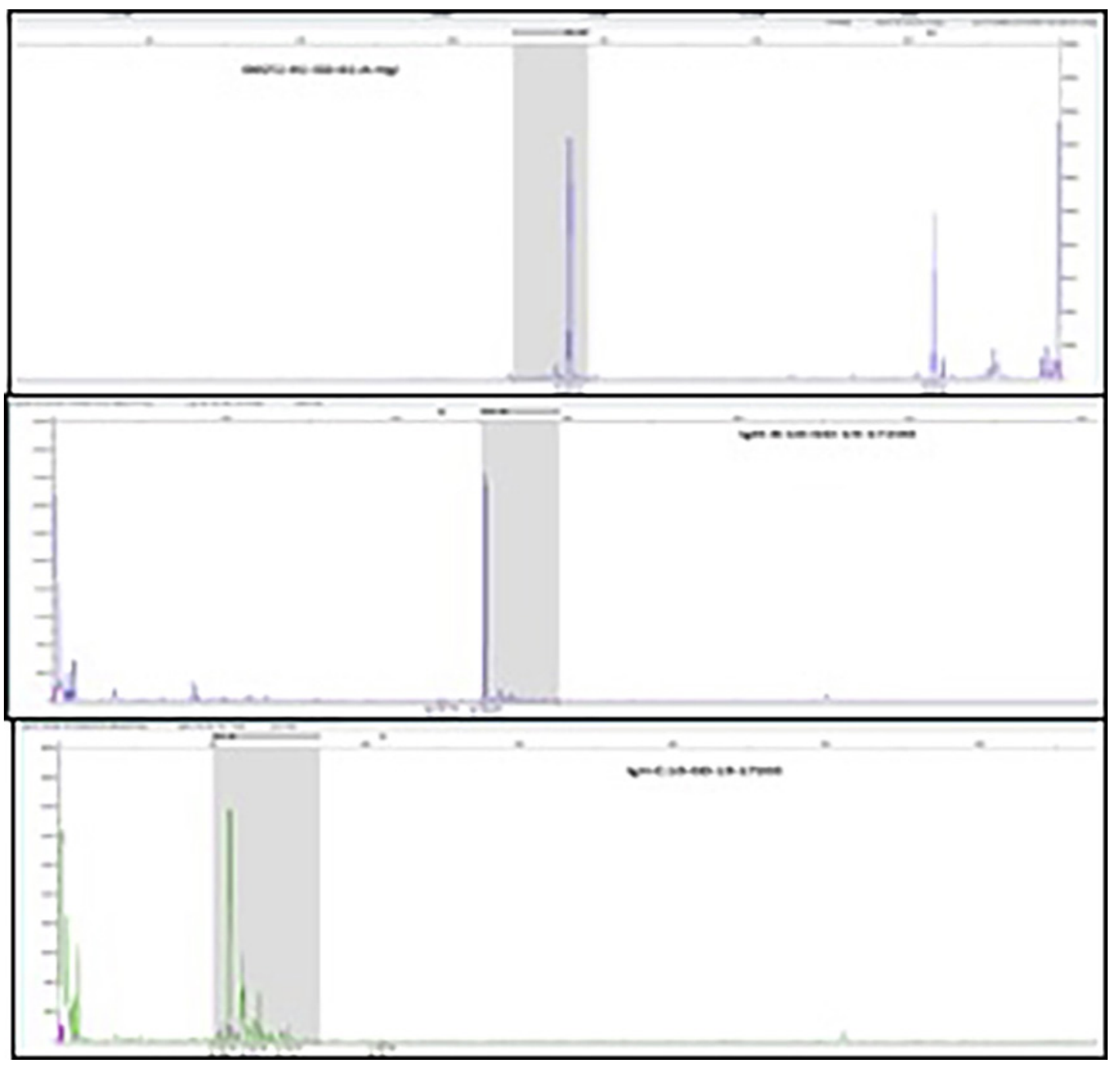

Fig. 4. Three tubes of fluorescently labeled primers that target the conserved framework (FR) of the immunoglobulin gene variable region together with primers for the conserved joining (j) regions were used to identify B cells derived from a single cell by detecting the V-J gene rearrangements. The analysis can detect the majority of clonal B-cell populations and provides clinical utility with respect to identification of clonality in atypical lymphoproliferative disorders.

The patient was investigated for underlying MM, and no monoclonal band was detected by serum protein electrophoresis with negative Bence-Jones protein in urine. BM examination revealed less than $1 \%$ CD34-positive myeloblasts and $0.2 \%$ polyclonal plasma cells. PET/CT scan revealed right pleural effusion with consolidation showing mild uptake and a small pleural effusion with left lung atelectasis, which is likely related to the infectious process; no abnormal activity was detected elsewhere.

The pleural effusion and respiratory symptoms were justified by active TB infection and the presence of concomitant plasma cell neoplasm was not convincing from clinical, radiologic and biochemical point of views. After starting anti-tuberculous treatment, he improved remarkably, and was discharged a week later with a plan to be followed up by an infectious disease and hematology teams. On follow-up done 1 and 3 months after the latest presentation, the patient showed significant improvement of respiratory symptoms confirmed by 


\section{Case Reports in Oncology}

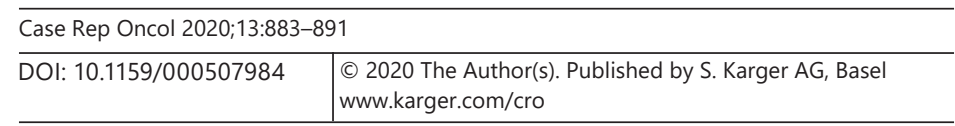

Sameh Soliman et al.: Transient Clonal Plasma Cell Associated with Pulmonary Tuberculosis

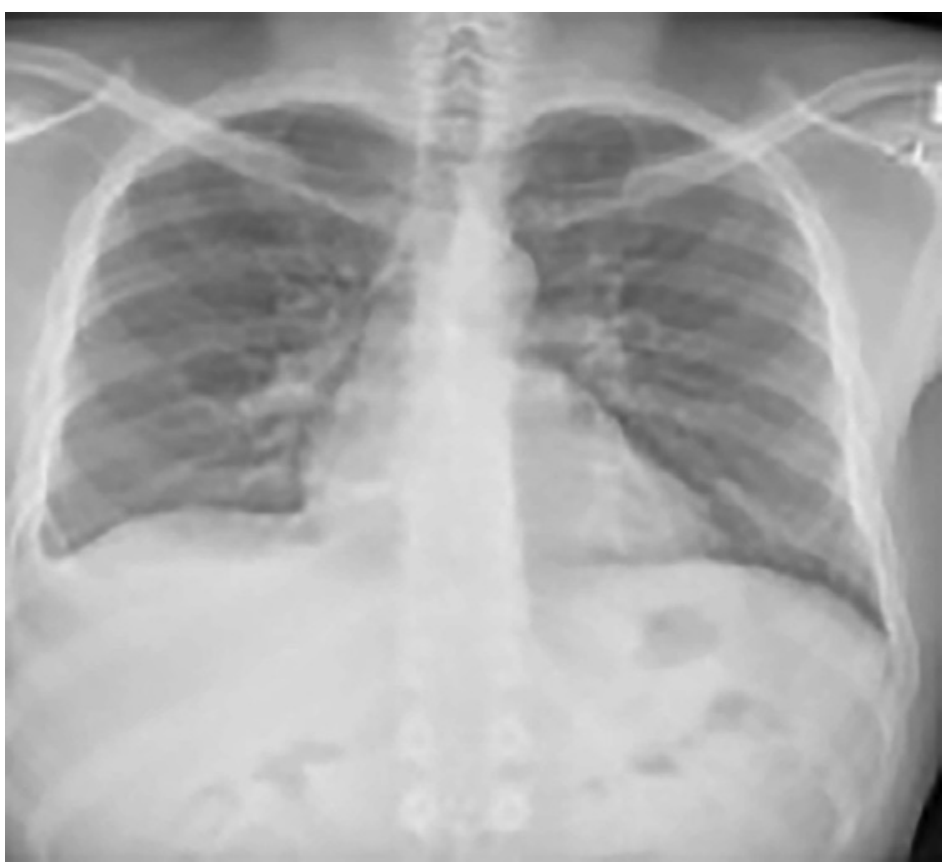

Fig. 5. Repeated chest X-ray 3 months after discharge showing significant resolution of right-sided pleural effusion.

repeated chest X-ray done on both visits revealing a significant resolution of pleural effusion (Fig. 5). It is planned for him to continue anti-tuberculous treatment for 6 months.

\section{Discussion}

The association between myeloid and plasma cell neoplasms is well established in the literature. In the great majority of patients, myeloid neoplasms developed following treatment of plasma cell neoplasms and were argued to be therapy related. Alkylating agents were postulated to be the culprit, and studies demonstrated an increased incidence of MDS and AML after using melphalan for treatment of MM [7]. Despite the fact that MM treatment has changed since the late 1990s with the introduction of high-dose therapy with autologous stem cell transplantation and the development of immunomodulatory agents, studies have shown an increased incidence of secondary malignancy in these patients, with a significant increased risk of AML reaching up to $9.8 \%$ at 9 years from first chemotherapy [7].

An intrinsic predisposition for both plasma and myeloid neoplasm has been addressed in the literature. However, coexistence of de novo MM and myeloid malignancies such as MDS, where no chemotherapeutic agents were given, are rarely reported in the literature [8].

There is no clear underlying pathophysiologic mechanism that explains the simultaneous occurrence of MM and myeloid neoplasm. One hypothesis is that MM evolves slowly from MGUS, which will lead to chromosomal instability and defect in the immune system with the inability to destroy evolving clones of malignant cells leading to the development of leukemia and MDS [8]. Another suggested mechanism is that the MM will lead to the activation of numerous gene expression profiles and molecular pathways leading to the formation of myeloid neoplasm. A recently published case report described the transdifferentiation of myeloid sarcoma from PCM, where next-generation sequencing demonstrated a clonal relationship between the PCM and transferred tumor cells, including loss of tumor suppressor gene, and multiple clonal mutations in the RAS pathway $[8,9]$.

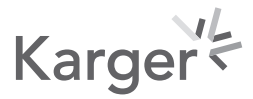


Sameh Soliman et al.: Transient Clonal Plasma Cell Associated with Pulmonary Tuberculosis

In our patient, the underlying background of AML followed by the detection of extramedullary clonal plasma cells in the pleural fluid raised the suspicion of an evolving PCM in a genetically predisposed patient. Pleural effusion is an unusual presentation of PCM, occurring in around $6 \%$ of patients, usually due to a concurrent disease process during the course of the disease [10]. Cardiac failure due to amyloidosis has been the most common cause for effusion in patients with PCM. A rarer mechanism occurring in less than $1 \%$ of the patients is the infiltration of pleural fluid by malignant plasma cells or direct infiltration of the pleural fluid from adjacent tissues leading to malignant MPE [6].

It is rare for patients with underlying PCM to present initially with MPE. In a Hispanic systemic review among the characteristics of 153 patients with MPE, few patients had MPE at first diagnosis of MM. MPE was predominantly unilateral (63.9\%) and usually involved more than two-third of hemithorax (54.5\%). In most cases of MPE, the pleural fluid profile is lymphocytic (78.6\%) and exudate $(97.4 \%)$ [11,12]. Upon literature review of English case reports about pleural effusion and MM, there were 17 cases of MPE as initial presentation of MM. All of the patients who presented initially with MPE had exudative pleural effusion and 14 of them had unilateral pleural effusion on initial presentation. Six of the 17 patients involved had nodular-like thickening of pleural, 3 cases had rib lyric lesions, and 2 patients had spinal lyric lesions combined with mediastinal abnormalities. The overwhelming majority had osteolytic lesions (12/15) proved by either X-ray or SP-ECT and at the time of diagnosis. Nearly all of the patients $(16 / 17)$ had associated pleural or chest wall plasmacytomas [5].

Diagnostic criteria to confirm the myelomatous etiology of pleural effusion include the detection of atypical plasma cells in pleural fluid cytology, demonstration of the monoclonal protein in pleural fluid electrophoresis (identical to serum protein electrophoresis), and histologic confirmation with pleural biopsy [6]. Our patient, however, did not fit the diagnostic criteria for plasma cell neoplasm as serum/urine protein electrophoresis, BM examination was negative and no evidence of plasmacytomas elsewhere was found. Beside the fact that PET-CT was unremarkable, it specifically did not show any sign of infiltration of the pleural membrane with malignant cells, and it did not reveal increased uptake in any adjacent tissue to suggest direct infiltration into the pleural fluid.

It is well known for TB to be associated with polyclonal gammaglobulinemia, but an association between TB and MGUS is not well established in the literature [3]. Upon literature review, Cheung et al. [13] reported a case of MGUS in a patient diagnosed with disseminated TB. Also, another case report demonstrated the coexistence of MGUS with TB in a patient with penile TB [14]. In the case described here, there is a clonal plasma cell proliferation, however, without any evidence of an underlying MGUS or PCM. In our patient, an extensive workup for an underlying PCM was negative initially and upon follow-up, and the origin of clonal plasma cells was unknown. Given all available evidence supported that the localized extramedullary (pleural) plasma cell proliferation probably represents a transient reactive process to pulmonary TB, which is an extremely rare phenomenon. To the best of our knowledge, this is the first report describing transient clonal extramedullary plasma cell proliferation in response to infectious process.

\section{Conclusion}

The association between myeloid and plasma cell neoplasms is mostly therapy related, and in the great majority of patients, myeloid neoplasms developed following treatment of plasma cell neoplasms. We described the formation of monoclonal plasma cells in pleural fluid of a patient known to have AML complicated by TB infection. We postulated that this 
might be the earliest manifestation of a plasma cell neoplasm in a genetically predisposed patient; however, extensive investigation and follow-up excluded this possibility.

A more likely hypothesis is that monoclonal plasma cells developed in the pleural fluid as a transient reactive response to pulmonary TB. It is well known for TB to be associated with polyclonal gammaglobulinemia. However, there are few cases that described MGUS in association with TB infection. To the best of our knowledge, the formation of extramedullary monoclonal plasma cells in the pleural fluid as a reactive inflammatory response to pulmonary TB was not described before in the literature.

The finding of monoclonal plasma cells in the pleural fluid in this patient was an incidental finding and probably would have been overlooked as flow cytometry is not routinely done on body fluids, particularly in the presence of infectious background. We believe this is an important observation and further data about extramedullary clonal plasma cell in patients with TB infection will help to establish and justify this association, which will spare the cost and burden of screening and monitoring patients with co-existent TB and similar findings for underlying plasma cell neoplasm.

\section{Acknowledgment}

The authors would like to acknowledge the Internal Medicine and Hematology Department at Hamad Medical Corporation for publication.

\section{Statement of Ethics}

Written informed consent was obtained from the patient to allow publication of information including images. The case was approved by HMC Medical Research Center.

\section{Conflict of Interest Statement}

The authors report no conflicts of interest in this work.

\section{Funding Sources}

No external funding provided.

\section{Author Contributions}

Dr. Mohammad Ali: concept, literature review, and manuscript writing.

Dr. Dina Soliman: concept, literature review, and manuscript writing.

Dr. Halima El Omri: concept, literature review, and manuscript editing.

Dr. Susanna Akiki, Dr. Feryal Ibrahim, Dr. Ahmad Al-Sabbagh, and Dr. Lina Okar: data and images collection, and manuscript editing. 


\section{Case Reports in Oncology}

\begin{tabular}{l|l}
\hline Case Rep Oncol 2020;13:883-891 \\
\hline DOI: 10.1159/000507984 & $\begin{array}{l}\text { ○ 2020 The Author(s). Published by S. Karger AG, Basel } \\
\text { www.karger.com/cro }\end{array}$ \\
\hline
\end{tabular}

Sameh Soliman et al.: Transient Clonal Plasma Cell Associated with Pulmonary Tuberculosis

\section{References}

1 Siegel RL, Fedewa SA, Miller KD, Goding-Sauer A, Pinheiro PS, Martinez-Tyson D, et al. Cancer statistics for Hispanics/Latinos, 2015. CA Cancer J Clin. 2020;65(6):457-80.

2 Rajkumar SV, Dimopoulos MA, Palumbo A, Blade J, Merlini G, Mateos MV, et al. International Myeloma Working Group updated criteria for the diagnosis of multiple myeloma. Lancet Oncol. 2014;15(12):e538-48.

3 Bida JP, Kyle RA, Therneau TM, Melton LJ 3rd, Plevak MF, Larson DR, et al. Disease associations with monoclonal gammopathy of undetermined significance: a population-based study of 17,398 patients. Mayo Clin Proc. 2009;84(8):685-93.

4 Landgren 0, Polliack A, Tadmor T. Associations between multiple myeloma and other malignancies. Leuk Lymphoma. 2011;52(2):161-2.

5 Zhang LL, Li YY, Hu CP, Yang HP. Myelomatous pleural effusion as an initial sign of multiple myeloma-a case report and review of literature. J Thorac Dis. 2014;6(7):E152-9.

6 Babu KA, Sundararajan L, Prabu P, Parameswaran A. Disseminated plasma cell myeloma presenting as massive pleural effusion. Eur Clin Respir J. 2015;2(1):27028.

7 Chung A, Liedtke M. Therapy-related myeloid neoplasms after treatment for plasma-cell disorders. Best Pract Res Clin Haematol. 2019;32(1):54-64.

8 Gajendra S. Clinics in oncology. Clin Oncol. 2016;1(1166):1-5. http://www.clinicsinoncology.com/ september-2017.php.

9 GralewskiJH, Post GR, van Rhee F, Yuan Y. Myeloid transformation of plasma cell myeloma: Molecular evidence of clonal evolution revealed by next generation sequencing. Diagn Pathol. 2018;13(1):15-7.

10 Cho YU, Chi HS, Park CJ, Jang S, Seo EJ, Suh C. Myelomatous pleural effusion: A case series in a single institution and literature review. Korean J Lab Med. 2011;31(4):225-30.

11 Arunsurat I, Reechaipichitkul W, So-Ngern A, Ungareevittaya P, Chaisuriya N, Teawtrakul N, et al. Massive pleural effusion due to IgG-Kappa subtype multiple myeloma: A case report. Respir Med Case Rep. 2020;29: 100984.

12 Riveiro V, Ferreiro L, Toubes ME, Lama A, Álvarez-Dobaño JM, Valdés L. Characteristics of patients with myelomatous pleural effusion. A systematic review. Rev Clin Esp.2018;218(2):89-97.

13 Cheung WI, Leung VK, Luk IS, Loke TK, Chan JC, Chau TN. Splenic tuberculosis associated with monoclonal gammopathy of undetermined significance and pericarditis. Hong Kong Med J. 2009;15(4):291-3.

14 Wu WC, Chen SC, Hsieh JT, Chen J, Chang HC. Penile tuberculosis associated with monoclonal gammopathy of undetermined significance. J Formos Med Assoc. 2006;105(9):753-5. 